\title{
Evaluación de fresas de diamante a diferentes métodos de esterilización
}

\author{
Arellano-Flores Alejandra**, Romo-Castillo Denise Sarai*, Flores-Maldonado Aldrín Noé***, Flores- \\ Arriaga Juan Carlos*, Gaitán-Fonseca César Iván*, Reyes Vela Enrique*, Goshima Kenichi***, \\ Masuoka-Ito David*
}

\begin{abstract}
- Resumen
- Es indispensable el evitar la contaminación cruzada en el con-

- sultorio dental. Existe poca información sobre los efectos de

- diferentes métodos de esterilización cíclicos sobre la eficacia

- de los instrumentos de corte de diamante. Cuatro grupos de

- cinco fresas de diamante se esterilizaron por cuatro métodos:

- esterilización con un agente químico, vapor a presión (auto-

- clave), calor seco, y vapor químico. Cada grupo de fresas de

- diamante fue sometido a los mismos ciclos de corte en un

- bloque cerámico para determinar la eficacia de corte. Todas

- las fresas se limpiaron por medio de ultrasonido en cada fase,

- se esterilizaron por cada método dependiendo de su grupo,

- y pasaron a la fase de corte. Al final de la repetición de 10

- ciclos completos, se observo que no había ninguna diferencia

- en la eficiencia del corte. Sin embargo, se encontró que exis-

- ten diferencias individuales en la eficacia de corte de cada

- uno de las fresas de diamante. La evaluación realizada en

- el microscopio electrónico de barrido (MEB) hecha antes del

- corte y al final de los 10 ciclos de esterilización demostró que

- el desgaste de diamante fue similar en todos los grupos y

- que la pequeña pérdida de partículas de diamante se dio de

- igual manera para todos los grupos. LUX MÉdICA AÑO 8, NÚMERO

- 23, ENERO-ABRIL 2013, PP 3-9
\end{abstract}

Palabras Clave: Fresas de diamante, esterilización, eficacia de corte

\section{Abstract}

It is essential to prevent the cross-contamination in the dental office. There is little information on the effects of different methods of cyclic sterilization on the effectiveness of the instruments cut of diamond. Four groups of five diamond burs were sterilized by four methods: sterilization with steam pressure (autoclave) and dry heat, chemical vapor, a chemical agent. Each group of diamond burs was subjected to the same cycles of cutting in a ceramic block to determine the effectiveness of court. All of diamond burs were cleaned using ultrasound in each phase, sterilized by each method depending on the group, and passed to the cutting stage. At the end of the 10 complete cycles, it was observed that no difference in the efficiency of the court there was. However, found that there are individual differences in the efficiency of cutting each diamond burs. The assessment in the MEB made before the court and at the end of the 10 sterilization cycles proved that diamond wear was similar in all groups, and that small loss of diamond particles occurred in the same way for all the groups. LUX MÉDICA AÑO 8, NÚMERO 23, ENERO-ABRIL 2013, PP 3-9

* Profesor Investigador del Departamento de Estomatología del Centro de Ciencias de la Salud de la Universidad Autónoma de Aguascalientes

** Alumnos de pregrado, carrera de Estomatología, Centro de Ciencias de la Salud, Universidad Autónoma de Aguascalientes

*** Alumno de posgrado del Centro de Ciencias de la Salud, Universidad Autónoma de Aguascalientes

**** Investigador, Universidad Médico Dental de Tokio, Escuela de Posgrado, Departamento de Prótesis Dental

Fecha de recibido: 1 de agosto 2012

Fecha de aceptación: 15 de enero 2013

Correspondencia: Dr David Masuoka Ito, Departamento de Estomatología, Centro de Ciencias de la Salud, Universidad Autónoma de Aguascalientes. Av. Aguascalientes No.940, Unidad Médico Didáctica, Edif.101, Ciudad Universitaria CP20131, Aguascalientes, Ags. México. Tel: +52 (449) 910 8438. Correo electrónico david.masuoka@gmail.com 


\section{Introducción}

Los procedimientos de control de infecciones han evolucionado y se han intensificado para evitar la contaminación cruzada en el consultorio dental. Los instrumentos dentales rotatorios de corte o fresas dentales, tienen la función de cortar o desgastar las superficies de los órganos dentales principalmente, por lo que están expuestos a la saliva, la sangre y los tejidos orales, por lo que su esterilización después de cada uso es indispensable. Se han cultivado microorganismos en placas de agar a partir de fresas dentales contaminadas. ${ }^{1}$ Además, existe evidencia donde se ha asociado la contaminación cruzada de la hepatitis B con los procedimientos dentales., 3

La calidad de corte de las fresas de diamante dentales es sumamente importante y existen muy pocos trabajos donde se estudien los efectos que sufren a través de las múltiples esterilizaciones. En ocasiones se ha cuestionado si la repetición de la esterilización de estos instrumentos puede disminuir su eficacia de corte al afectar a la matriz donde se unen partículas de diamante al vástago, produciendo la pérdida de las partículas de diamante. Para mantener la eficacia de desgaste de una fresa de diamante, Harkness y Davies reportaron que los instrumentos deben ser limpiados por ultrasonido, porque las soluciones de limpieza con un $\mathrm{pH}$ mayor que 11 pueden ocasionar la corrosión de la matriz de níquel, lo que contribuye a la pérdida de partículas de diamante y la disminución de la eficacia de desgaste. ${ }^{4-7}$ Por otro lado, Hooker y Staffanou en su estudio observaron que los instrumentos de diamante al ser sometidos a la limpieza por ultrasonidos y autoclave, presentaron una pequeña pérdida de partículas de diamante. ${ }^{7}$ Aarli y Myklebus reportaron los efectos de desgaste y la corrosión de los instrumentos de diamante en autoclave, al realizar este procedimiento 15 veces y posteriormente ser utilizadas para la preparación del órgano dentario. $^{7-9}$

Las directrices actuales recomiendan el control de infecciones de los instrumentos de corte rotatorios hechos de acero inoxidable o carburo por medio de esterilización con el autoclave, horno de calor seco, vapor químico, óxido de etileno, y agentes químicos. ${ }^{10} \mathrm{Re}-$ cientemente, los agentes químicos utilizados para la esterilización en frío se han hecho populares, ya que son económicos y fáciles de utilizar. Sin embargo, estos agentes requieren de largos tiempos de exposición para destruir efectivamente todos los microorganismos y esta exposición prolongada puede producir la corrosión de los instrumentos.

Hasta la fecha, existen muy pocos estudios comparativos sobre los efectos de diferentes métodos de esterilización cíclicos sobre la eficacia de los instrumentos de corte de diamante. Este estudio evaluó la eficacia de desgaste de las fresas de diamante dentales esterilizados repetidamente por cuatro métodos diferentes. 


\section{Material y métodos}

Veinte fresas de diamante nuevas con punta redondeada de grano grueso TwoStriper No 770.8C (Abrasive technology, Ohio, EEUU) del mismo número y lote fueron seleccionadas para el estudio. Cada una fue examinada bajo un microscopio estereoscópico 30x para observar sus características, la distribución del diamante y la extensión de la matriz. Las fresas de diamante que presentaron algún problema en los puntos anteriores fueron eliminadas. Se evaluaron los cuatro métodos de esterilización por medio de cuatro grupos que constaron de cinco fresas de diamante seleccionadas al azar (Tabla I).

Los dispositivos de esterilización fueron calibrados para asegurar que la temperatura recomendada y la presión para la esterilización se llevara a cabo. Se utilizaron soluciones nuevas de glutaraldehído al 2\%, Sporicidin (Sporicidin Co., Washington DC, EEUU), para cada fase de esterilización en frío. En el caso del vapor químico se utilizó la solución Harvey's Vapo-Steril Solution (MDT Biologic Company Nueva York, EEUU). Los detalles de los métodos de esterilización se describen en la Tabla 1.

\section{Tabla I}

\begin{tabular}{|c|c|}
\hline Método & Características del procedimiento \\
\hline Esterilización en Frío ${ }^{a}$ & 6.75 hrs. En $2 \%$ de glutaraldehído (Sporicidin) \\
\hline Vapor Químico (Chemiclave) ${ }^{\mathrm{b}}$ & 20 min. A $132^{\circ} \mathrm{C}$ a 20 psi. \\
\hline Vapor Presión (Autoclave) c & $15 \mathrm{~min} . \mathrm{A} 121^{\circ} \mathrm{C}$ a $15 \mathrm{psi}$ \\
\hline Calor Seco ${ }^{d}$ & $1 \mathrm{hr} . \mathrm{A} 170^{\circ} \mathrm{C}$ \\
\hline
\end{tabular}

Descripción de los métodos de esterilización. a Sporicidin Company, Washington, D.C., EEUU b MDT Corp. California, EEUU c AMSCO General Purpose, American Sterilizer Co., Pensilvania, EEUU d Gruenberg Oven Co., Inc., Pensilvania, EEUU.

La eficacia de corte se evaluó con un motor de aire de precisión Planet 2000 (NSK-America Corp., Illinois, EEUU) con una velocidad de funcionamiento libre de 187,000 rpm a 35psi. El material de corte fueron bloques de cerámica de vidrio de $1.5 \mathrm{~cm} . \times 1.5 \mathrm{~cm} . \times 8 \mathrm{~cm}$. MACOR (Machinable Glass-Ceramic, Nueva York, EEUU), las cuales son comúnmente utilizadas por algunos fabricantes para la prueba de fresas dentales. Este material tiene una escala de dureza Knoop similar a la del esmalte dental. ${ }^{11}$ Cada bloque MACOR fue unido a una plataforma móvil, la cual se tira horizontalmente con una carga constante de $140 \mathrm{~g}$. Contra el instrumento giratorio de diamante o fresa, con una velocidad promedio de corte de 182,600 rpm. La refrigeración se realiza con agua la cual se proporcionan a través de una línea que irriga $20 \mathrm{~cm}^{3} / \mathrm{min}$. En la zona de corte para evitar la obstrucción y atascamiento de la fresa.

Para medir la eficacia de corte, se llevó a cabo un corte inicial o de entrada en el bloque MACOR con cada instrumento de diamante sin usar. Este corte se utilizó como control para las variaciones inherentes en la construcción del instrumento. Antes de la esterilización, cada fresa se colocó en el motor de aire de precisión, y se comenzó a cortar el bloque MACOR durante 5 minutos. La profundidad del corte fue estandarizado para todas las fresas. La longitud del corte se midió en milímetros posteriormente mediante el uso de un dispositivo electrónico de pinza (MAX-CAL, Ted Pella, Inc., California, EEUU).

Se retiraron las fresas del motor de aire, y se limpiaron por ultrasonido $(L+$ R Manufacturing Company, Nueva Jersey, EEUU) durante 10 minutos en agua desti- 
lada. Los instrumentos que iban a ser esterilizados con el vapor a presión, el vapor químico, y el calor seco, se secaron al aire y se colocaron en freseros termoplásticos (Thermo-Block, Blanco SS, Nueva Jersey, EEUU) para evitar la posibilidad de corrosión del metal. El grupo de instrumentos de diamante a ser esterilizado con vapor químico y vapor a presión se envasa en tubos de esterilización de nylon y doble sellado térmico. El fresero del grupo de calor seco se colocó en una bolsa de papel de esterilización antes de la misma. La esterilización se controló con indicadores químicos colocados en cada envase para verificar la esterilización. Los instrumentos de diamante en el grupo de esterilización en frío se coloca- ron en recipientes individuales de plástico que contienen soluciones nuevas de Sporicidin durante 6.75 horas, y se enjuagaron en agua destilada antes de la prueba. Este patrón de pruebas de limpieza ultrasónica, y la esterilización se llevó a cabo durante 10 ciclos. Se utilizó un análisis de varianza (ANOVA) para evaluar los datos sobre la eficacia de corte. El nivel $p=0,05$ fue utilizado para determinar la significancia.

Las alteraciones superficiales de las fresas se evaluaron con un microscopio electrónico de barrido (MEB) al inicio y al final de los ciclos de la prueba. Las muestras fueron montadas sobre bases de aluminio y fueron observadas con 75 y 150 aumentos de potencia.

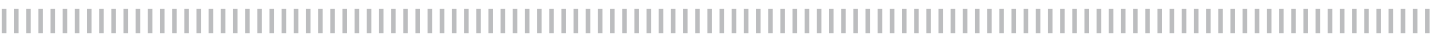

\section{Resultados}

La eficacia de corte de los instrumentos de diamante no fue influenciado por el método de esterilización. Los efectos de los ciclos de esterilización sobre la eficacia de corte se presentan gráficamente en las figuras 1 y 2. Cada barra representa el promedio del corte o reducción media de 5 minutos realizado en el bloque MACOR para las cinco fresas de cada número de ciclo. La variación entre las fresas con el primer corte o control hace hincapié en las diferencias inherentes entre cada fresa, incluso siendo del mismo tamaño, forma y tamaño de partícula. En el octavo corte de prueba, sin embargo, la mayoría de los instrumentos de diamante exhibieron rangos de corte similares. El grupo de esterilización química mostró una mejor tendencia al corte, principalmente durante la $6^{a}$ y $7^{a}$ prueba, que fue disminuyendo hasta un patrón similar al de los otros grupos de esterilización en la octava prueba.

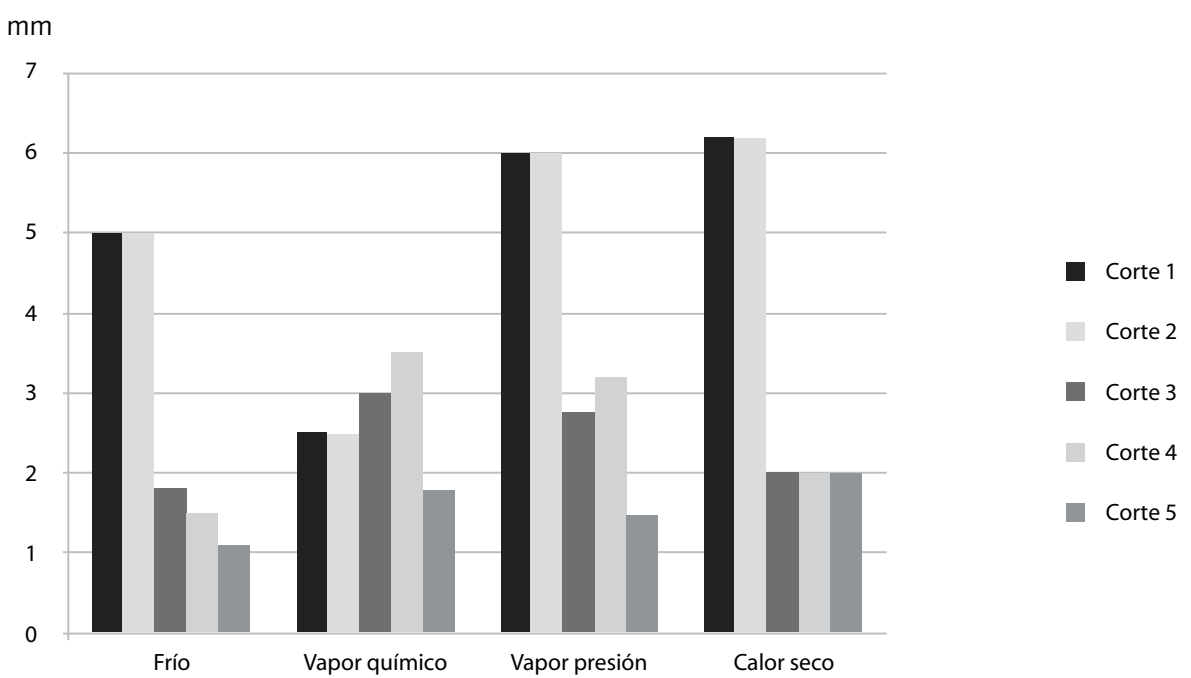

Fig. 1 Promedio de longitud de corte de las cinco fresas de diamante en cada grupo para los cliclos 1 a 5. Los datos numéricos de la parte izquierda representa la longitud de corte en el bloque MACOR (mm). 


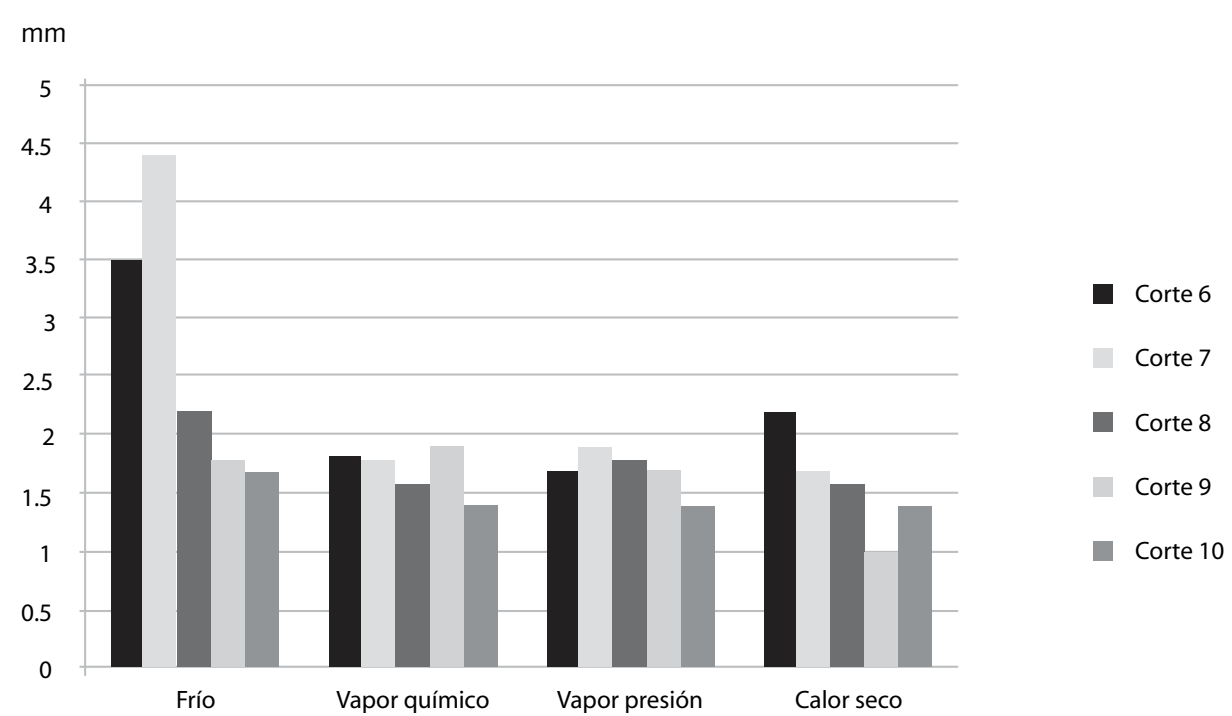

Fig. 2 Promedio de longitud de corte de las cinco fresas de diamante en cada grupo para los cliclos 1 a 5. Los datos numéricos de la parte izquierda representa la longitud de corte en el bloque MACOR ( $\mathrm{mm})$.

Las observaciones en el MEB con 75 y 150 aumentos de potencia de la fresa sin utilizar, muestra que las partículas de diamante están uniformemente espaciadas, y el material de la matriz se superpone sobre cada partícula. Al realizar una comparación de las microfotografías de las fresas no utilizadas y de las fresas de los cuatro grupos de esterilización, después de los 10 cortes de prueba y sus respectivos ciclos de esterilizaciones, se encontraron diversas cantidades de partículas de diamante aplanadas y desgastadas para todos los grupos. Sin embargo, se pudieron apreciar pocas señales de pérdida real de partículas de diamante. Se observó que el grupo de esterilización en frío tenía menos desechos en la superficie de los instrumentos de diamante que los otros tres grupos, a pesar de que cada grupo se sometió a la limpieza ultrasónica antes de la evaluación en el MEB. Esta condición puede ser debido a un efecto o disolución química de los desechos por la solución de esterilización en frío. La textura de la superficie del material de la matriz del grupo de vapor químico mostró menos detalle de la superficie (menos estrías) que la de los otros grupos.

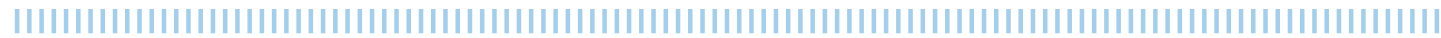

\section{Discusión}

No se produjo una diferencia en la eficacia de corte de fresas de diamante al ser sometidas por 10 ciclos de esterilización con el agente químico (Sporicidin), con vapor a presión (autoclave), con calor seco, o con vapor químico.

Aunque hay algunas especificaciones y criterios de los protocolos para pruebas de instrumentos rotatorios de diamante en cuanto a las formas, la cabeza, y las dimensiones de vástago en la estandarización No.
36 para instrumentos rotativos de diamante de la Asociación Dental Americana, no hay especificaciones en cuanto a la fuerza, la eficiencia del corte, la durabilidad, la cantidad, tamaño y tipo de partícula de diamante.

Nos enfocamos en controlar el mayor número de variables posible (profundidad de corte, la duración del corte, carga, velocidad de irrigación del agua, y las revoluciones por minuto). Sin embargo, hubieron variables inherentes a este dispositivo de 
prueba que no pudo ser totalmente controlado. Un motor de aire de precisión se utiliza en lugar de una pieza de mano de alta velocidad, ya que el desgaste del rodamiento en la pieza de mano produciría descentramiento y velocidades reducidas, afectando negativamente la eficiencia del instrumento de corte. Utilizando una pieza de mano de alta velocidad, Johnson y cols $^{12}$, informaron de una disminución del $14 \%$ en las revoluciones por minuto de la pieza de mano al final de un estudio. Por este motivo se optó por utilizar el motor de aire de precisión, ya que controla esta variable y produce revoluciones por minuto similar a las revoluciones por minuto en las piezas de mano dentales.

Varios materiales como sustratos de corte se han utilizado, como son el vidrio, latón, diente, cerámica, hierro fundido, marfil y hueso, obteniendo resultados variables. Grajower y cols. ${ }^{13}$ recomiendan el uso de un material plano, de espesor, propiedades físicas, y contenido de humedad uniformes. Hartley et al. ${ }^{14}$ recomendaron el vidrio para determinar la resistencia a la pérdida de abrasividad, y un material diferente que no ocasione ruptura para la determinación del grado de corte. Gegauff y cols. ${ }^{15}$ probaron varios materiales de sustrato y reportaron una importante gama de propiedades de corte. La cerámica de vidrio mecanizable MACOR fue seleccionada para este estudio por su utilidad en otros estudios, y debido a que fue recomendada por algunos fabricantes de fresa. Sin embargo, algunos materiales de sustrato en realidad puede "vestir" el instrumento e inadvertidamente quitar material de la matriz, exponiendo más superficies de las partículas de diamante, haciendo que las partículas de diamante sean más vulnerables a desalojarse.

El instrumento de diamante seleccionado para este estudio (Two-Striper No 770.8C) representa un instrumento de diamante ampliamente utilizado. Sólo se empleo un tipo y forma del mismo fabricante, ya que las variaciones de diámetro tan pequeño como $0.1 \mathrm{~mm}$ puede producir diferencias significativas en el rendimiento de corte Ting. ${ }^{16}$ El fabricante de este instrumento utiliza un proceso de soldadura directa para adherir las partículas de diamante en lugar de galvanoplastia, la cual es utilizada por muchas otras marcas. Sería inexacto correlacionar los resultados de este estudio con los de otros estudios que utilizan diferentes instrumentos de diamante.

La distribución de las partículas de diamante podrían afectar la eficacia de corte del instrumento. Westland ${ }^{17}$ observó que sólo unos pocos granos en un instrumento de diamante son los que en realidad están implicados en el corte en cualquier punto en el tiempo, y como las partículas de desgaste, otras partículas se activan en el proceso de corte. Green y Lindenmeyer ${ }^{18}$, así como, Reisbick y Bunshah ${ }^{19}$, describieron un modo de embotamiento casi lineal para las fresas de carburo, en el que un corte subsecuente presenta una mejor eficiencia de corte que el corte anterior. Los autores sugieren que esta mejora de la eficiencia de corte puede ser debido a la fractura de las cuchilla o dientes de corte ocasionadas durante su desgaste, lo que crea entonces un borde cortante agudo y más afilado. Esta mejora también podría ser planteada para los instrumentos de diamante, ya que las partículas de diamante durante su desgaste también sufren fracturas, exponiendo nuevas superficies agudas y afiladas. Este hallazgo puede explicar algunas de las mejoras transitorias en la eficacia de corte observada en este estudio.

Harkness y Davies ${ }^{4}$ concluyeron que una solución de esterilización en frío con un $\mathrm{pH}$ alto tendría un efecto perjudicial sobre el componente de níquel que conforma el material de la matriz, lo que conduciría a disminuir el soporte para las partículas de diamante y la picadura de la superficie. Las observaciones en el MEB no revelaron cambios en la matriz causada por el elevado $\mathrm{pH}$ del Sporicidin. Los instrumentos de diamante expuestos a vapores químicos presentaron una matríz con superficie 
lisa, pero sin diferencia en la relación de la matriz y las partícula de diamante. Los resultados de este estudio mostraron efectos insignificantes de esta marca de solución sobre el material de la matriz y el instrumento de diamante como tal.

Una limpieza adecuada de los instrumentos de corte rotatorios previo a la fase de la esterilización, es un paso importante en el proceso de esterilización y reducción de la eficacia del instrumento. ${ }^{4,6-8}$ La presencia de restos (sangre, suero, mucosa, etc.), puede hacer que todos los métodos prácticos de esterilización o desinfección sean ineficaces en su totalidad. Las proteínas presente en fresas dentales reducen significativamente el efecto bactericida de las soluciones alcalinas del glutaraldehído. ${ }^{2}$ Adicionalmente, los instrumentos obstruidos con restos reducen la efectividad de corte. ${ }^{13}$ Cabe mencionar, que incluso con la irrigación de agua de $20 \mathrm{~cm}^{3} / \mathrm{min}$ utilizado en este estudio, el material de sustrato se adhirió al instrumento, por lo que la limpieza ultrasónica se sugiere para reducir considerablemente los restos del sustrato.

\section{Conclusiones}

1. La eficacia de corte de las fresas de diamante no fue influenciado por el método de esterilización.

2. Hay diferencias considerables en la eficiencia de corte de cada fresa de diamante nueva.

3. Las observaciones por medio del MEB demostraron que el desgaste del diamante fue similar en todos los grupos y hubo poca pérdida de partículas de diamante en cada grupo.

\section{Bibliografía}

1. Cooley RL, Barkmeir WW, Wayman BE. Sterilization and disinfection of dental bur. Gen Dent 1982;30:608-12.

2. Neugeboran $N$, Nisengard RJ, Beutner EH, Ferguson GW. Control of cross-contamination. J Am Dent Assoc 1972;88:123-7.

3. Sanger RG, Bradford BA, Delaney JM. An inquiry into the sterilization of dental handpieces relative to transmission of hepatitis B virus. J Am Dent Assoc1978;96:621-4.

4. Harkness N, Davies EH. The cleaning of dental diamond burs. Br Dent J 1983;154:42-5.

5. Hegde J, Sravanthi Y. An in vitro comparison of adhesive techniques and rotary instrumentation on shear bond strength of nanocomposite with simulated pulpal pressure. J Conserv Dent. 2011;14(3):228-32.

6. Dong G, Ren LB, Lü YY, Ye L, Li JY, Tan H. Effect of different sterilization methods on mechanical properties of dental corrosi bureffects of different sterilization methods on mechanical properties of dental corrosi bur. Hua Xi Kou Qiang Yi Xue Za Zhi. 2009;27(2)199-201-5.

7. Li RM, Wang $C L$, Xu GX, Ye L, Tan H. Effect of different sterilization methods on the corrosion of dental fissure bur. Hua Xi Kou Qiang Yi Xue Za Zhi. 2007;25(6):580-3.

8. Hooker JB, Staffanou RS. An evaluation of sterilization procedures of diamond cutting instruments. Texas Dent J 1985;103:8-10.
9. Aarli VG, Myklebust S. Desinfeksjon og starilisaring av dentale stalog diiantbor. Den Nokske Tannlegeforeniugs Tidende 1986;96:6096-12.

10. Council on Dental Materials, Instruments, and Equipment. Infection control recommendations for the dental office and the dental laboratory. J Am Dent Assoc 1988;16:241-8.

11. Watanabe $L$, Soelberg KB, Pelzner RB, et al. Diamond stones. Rep Product eval Lab 1987;3:17-23.

12. Johnson GK, Perry FU, Pelleu GB. Effect of four anticorrosive dips on the cutting efficiency of dental carbide bur. J Am Dent Assoc 1987; 114:648-50.

13. Grajower R, Zeitchick A, Rajstein J. The grinding efficiency of diamond burs. J Prosthet Dent 1979;42:422-8.

14. Hartley JL, Hudson DC, SweeneyWT, Dickson G. Methods for evaluation of rotating diamond-abrasive dental instruments. J Am Dent Assoc 1957;54:637-44.

15. Gegauff A, Rosensteil S, Bleiholder R, Martello J, McCafferty R. Substrate differences in rotary instrument testing [Abstract]. J Dent Res 1989;68:400.

16. EamesWB, Reder BS, Smith GA. Cutting efficiency of diamond stones: effect of technique variables. Oper Dent 1977;2:156-64.

17. Westland IN. The energy requirement of the dental cutting process. J Oral Rehabil 1980;7:51-63.

18. Greener EH, Lindenmeyer RS. Bur geometry and its relationship to cutting. J Dent Res 1968;47:87-97.

19. Reisbick MH, Bunshah RF. Wear characteristics of burs. J Dent Res 1973;52:1138-46. 